\title{
René Chapallaz. Architecte de la Tavannes Watch Co
}

\section{René Koelliker}

\section{OpenEdition}

Journals

Édition électronique

URL : http://journals.openedition.org/edl/234

DOI : $10.4000 /$ edl. 234

ISSN : 2296-5084

\section{Éditeur}

Université de Lausanne

\section{Édition imprimée}

Date de publication : 15 décembre 2010

Pagination : 103-122

ISBN : 978-2-940331-24-6

ISSN : 0014-2026

\section{Référence électronique}

René Koelliker, «René Chapallaz. Architecte de la Tavannes Watch Co », Études de lettres [En ligne], 4 I 2010, mis en ligne le 15 décembre 2013, consulté le 20 décembre 2020. URL : http:// journals.openedition.org/edl/234; DOI : https://doi.org/10.4000/edl.234 


\section{RENÉ CHAPALLAZ \\ ARCHITECTE DE LA TAVANNES WATCH CO}

En 1905, la Tavannes Watch Co emploie plus de six cents ouvriers et figure parmi les plus grandes fabriques d'horlogerie de Suisse. Cofondateur de l'entreprise, HenriFrédéric Sandoz (1851-1913), fort de ce succès, nourrit de grandes ambitions architecturales pour son entreprise et engage René Chapallaz (1881-1976), architecte établi à La Chaux-de-Fonds. La collaboration débute en automne 1905 et se termine en octobre 1907. En quelques mois, Chapallaz construit la villa de la famille Sandoz, un bureau d'architecture, un lotissement de maisons et une fabrique dont les éléments architecturaux et décoratifs sont largement influencés par le Heimatstil et le "style sapin" développé à l'Ecole d'art de La Chaux-de-Fonds.

Le village de Tavannes permet une intéressante lecture architecturale en lien avec le développement de l'industrie horlogère qui s'implante dans le Jura bernois à partir de la seconde moitié du XIX ${ }^{\mathrm{e}}$ siècle. D'un village essentiellement agricole, Tavannes devient, en quelques années, une petite cité "urbanisée» dans laquelle le patrimoine rural est progressivement remplacé par l'ambitieux programme architectural mis en place par la direction de la Tavannes Watch Co, principale industrie du lieu ${ }^{1}$. Fondée en 1895 par le fabricant Henri-Frédéric Sandoz, en collaboration et avec le soutien de la bourgeoisie du village ${ }^{2}$, instigatrice

I. Pour une connaissance plus approfondie de l'histoire de la Tavannes Watch Co nous renvoyons le lecteur à l'ouvrage de Ch. Gagnebin-Diacon, La fabrique et le village.

2. Il convient de préciser le terme bourgeoisie: il ne s'agit pas d'une élite économique, mais d'une assemblée composée des familles originaires du village de Tavannes. Dans de nombreuses communes du Jura bernois, il pouvait exister une assemblée de la bourgeoisie et une assemblée communale. Aujourd'hui, les assemblées de bourgeoisie se retrouvent pour gérer les biens qu'elles possèdent et n’ont plus de pouvoir politique. 
du projet, et financièrement soutenue par les sociétés Schwob Frères Co et Schwob Co implantées à La Chaux-de-Fonds, la Tavannes Watch Co devient en quelques années le principal employeur de la région et figure parmi les plus grandes entreprises horlogères du début du $\mathrm{XX}^{\mathrm{e}}$ siècle en Suisse. En 1905, elle occupe six cent neuf ouvriers ${ }^{3}$. Ce succès commercial influence directement le domaine de la construction et l'ambitieux patron de la Tavannes Watch Co prévoit non seulement le développement de ses affaires mais également la construction ou reconstruction de Tavannes afin d'accueillir les nombreux ouvriers qui travaillent pour lui. Entre 1890 et la Seconde Guerre mondiale sont élevées les fabriques de la Tavannes Watch Co et de la Tavannes Machines Co qui, de nos jours encore, marquent l'est du village de leurs imposantes silhouettes. De nouveaux quartiers de maisons locatives entourées de jardins potagers, des hôtels, des restaurants et des immeubles aux vocations commerciales le long de la rue principale et dans le périmètre de la gare, un cinémathéâtre, Le Royal, un Cercle démocratique et quelques villas patronales sortent de terre en quelques années. Au début du XXe siècle, il semble que Tavannes ne soit qu'un énorme chantier qui va considérablement transformer le paisible village à vocation rurale en une petite cité urbanisée. Les bâtiments construits au cours de cette période sont largement imprégnés d'éléments architecturaux éclectiques ou proches du Heimatstil, au niveau du traitement des façades, des toitures et du langage décoratif. Ce dernier est parfois influencé par l'Art nouveau international ou par sa version régionale, le "style sapin", développée à l'Ecole d'art de La Chaux-de-Fonds. Les étroits contacts avec la métropole horlogère des montagnes neuchâteloises ne sont pas étrangers au choix d'HenriFrédéric Sandoz, alors à la recherche d'un architecte, d'engager René Chapallaz afin de concrétiser le vaste programme architectural qu'il prévoit. La collaboration entre René Chapallaz et la Tavannes Watch Co débute en septembre 1905 et se termine de manière abrupte en octobre 1907. Malgré la courte durée de son séjour, Chapallaz construit un atelier d'architecture, un lotissement de maisons ouvrières, influencé par la cité-jardin, et une fabrique. Il suit également le chantier de la villa de la famille Sandoz décorée à l'intérieur par les élèves de l'Ecole d'art de La Chaux-de-Fonds. Pour perfectionner ses connaissances architecturales et

3. P.-Y. Donzé, Histoire de l'industrie horlogère suisse, de Jacques David à Nicolas Hayek (1850-2000), p. 48. 
sur demande de Sandoz, il entreprend un voyage d'étude dans le nord de l'Europe. Nous pouvons le constater, Chapallaz semble très actif et Sandoz apprécie son travail. Pourtant, en quelques mois, le climat se détériore et la rupture est la seule issue.

\section{Le client de l'architecte: une collaboration complexe}

La collaboration entre Henri-Frédéric Sandoz, le maître d'ouvrage, et René Chapallaz, l'architecte, est de nos jours encore empreinte de mystère. Chapallaz est-il un architecte indépendant avec un important client, Henri-Frédéric Sandoz? Ou est-il un "chef de chantier", rémunéré et dépendant de la direction de la Tavannes Watch Co? La première hypothèse semble se confirmer pour deux raisons: d'une part, la construction d'un bureau d'architecture qui appelle à l'indépendance (en effet, Chapallaz n'intègre pas un bureau au sein de la fabrique mais se construit une maison qui devait abriter son atelier d'architecture), d'autre part, Chapallaz collabore à l'élaboration de la villa Fallet à La Chauxde-Fonds en collaboration avec Charles-Edouard Jeanneret, le futur Le Corbusier, en 1906, alors qu'il est déjà à Tavannes. Ce statut particulier d'indépendant, si tel était le cas, a certainement dû mener à des conflits d'intérêts entre l'architecte et son client. De plus, les liens familiaux qui unissaient les deux hommes - Chapallaz épouse Rose, la fille cadette de son "patron" - ne devaient pas faciliter les rapports de travail. Ces différents paramètres relativement complexes ne pourraient-ils pas expliquer, en partie, l'origine du départ précipité de l'architecte vers La Chaux-deFonds en octobre 1907?

De nombreuses questions restent à ce jour sans réponses, mais estil important d'y répondre? N'est-il pas plus intéressant de présenter, à travers une biographie succincte, les deux principaux protagonistes de "l'aventure Chapallaz à Tavannes» et nous concentrer sur le patrimoine bâti qui en a résulté?

\section{Henri-Frédéric Sandoz (1851-1913), le maître d'ouvrage et fabricant}

Henri-Frédéric Sandoz naît au Locle le 5 mars 1851 (fig. 1). A l'âge de 13 ans, il entreprend un apprentissage au sein de l'atelier d'emboîtage de 
Fig. 1 - Henri-Frédéric Sandoz (à gauche) cofondateur de la Tavannes Watch Co et son fils Charles, vers 1907-1910. (Collection privée, Paris).

Samuel Aubert. En 1869, il se met à son compte comme emboîteur et fabricant de ressorts-timbres. Il prend très rapidement conscience du progrès de la production américaine qui travaille à la chaîne en employant la machine-outil pour fabriquer les pièces d'horlogerie. Il participe à l'Exposition universelle de Philadelphie de 1876. En 1880, à la recherche de terrain pour étendre ses activités, Sandoz se heurte à Georges FavreJacot, patron des montres Zénith, et doit donc émigrer pour pouvoir développer son savoir-faire. Il s'installe à Tavannes en 1891 et fonde, en collaboration avec d'autres acteurs, la Tavannes Watch Co. Il meurt dans ce village en 1913 en laissant un empire industriel et patrimonial d'une extrême richesse.

\section{René Chapallaz (1881-1976), l'architecte}

René Chapallaz naît à Nyon en 1881 (fig. 2). Dès 1897, il est apprenti à Zurich auprès du bureau Pfleghard \& Häfeli et y travaille ensuite deux ans comme dessinateur. En parallèle, il suit les cours de la Kunstgewerbeschule. Dans le cadre de son travail, il participe à la 


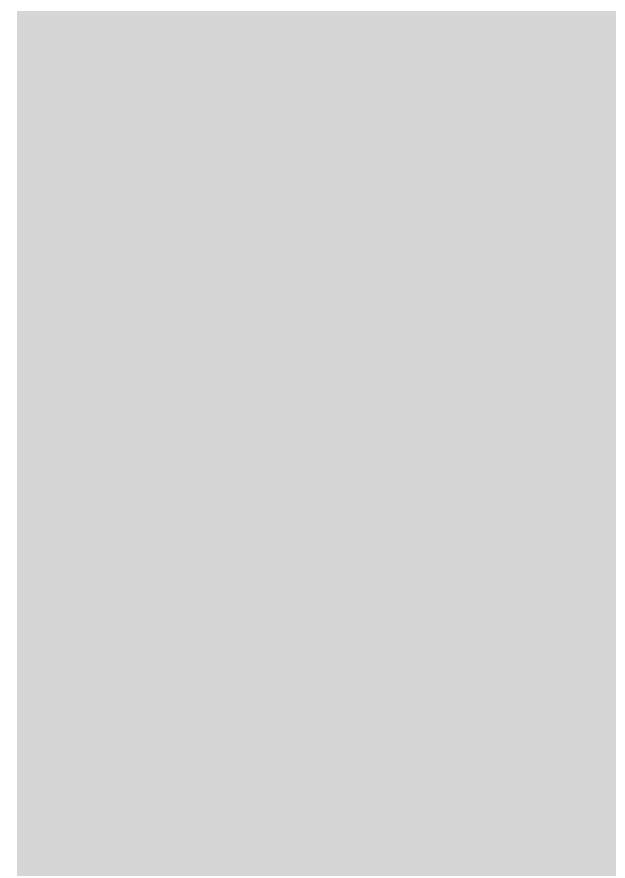

Fig. 2 - René Chapallaz, architecte, vers 1915.

(Bibliothèque de la ville de La Chaux-de-Fonds. Fonds René Chapallaz).

construction du «Schloss Utoquai» et de prestigieuses villas, dont la villa Grieder. Après un court passage à Genève, il est engagé en 1902 comme dessinateur-architecte, puis en tant que chef de travaux chez Edouard Piquet, architecte constructeur à La Chaux-de-Fonds. Il se lie d'amitié avec Charles L'Eplattenier et s'intéresse particulièrement au "style sapin" développé à l'Ecole d'art. Lors de son premier séjour dans la métropole des montagnes neuchâteloises, Chapallaz travaille à plusieurs projets : la villa de Charles L'Eplattenier en 1902, la villa Gallet en 1904 et enfin la villa Fallet en 1906, comme nous l'avons déjà mentionné. Entre 1905 et 1907, il est "architecte indépendant" à Tavannes. A partir de 1908, sa carrière se déroule presque exclusivement dans les montagnes neuchâteloises. Il est notamment l'auteur du Musée des beaux-arts, réalisé entre 1923 et 1925, de nombreuses fabriques d'horlogerie et de villas de fabricants. René Chapallaz est un passionné de photographies, curieux des nouvelles techniques du bâtiment et un membre assidu du Club alpin suisse. Il décède à La Chaux-de-Fonds en 1976. 


\section{Le patrimoine bâti de René Chapallaz à Tavannes}

A son arrivée à Tavannes, René Chapallaz se met très rapidement au travail. En quelques mois, il semble mener de front la construction de son atelier, la planification du lotissement de Sonrougeux et le suivi du chantier de la villa Sandoz, dont les plans ont été dessinés par les architectes Vuilleumier et Renk. Ses mandats sont interrompus par un voyage qu'il effectue au printemps 1906, que nous proposons de présenter plus tard. A son retour, Sonrougeux est réalisé et, à la même période, il collabore à la construction de la villa Fallet à La Chaux-de-Fonds et termine sa carrière à Tavannes par un bâtiment industriel. Les réalisations de Chapallaz sont empreintes de Heimatstil, dans le langage stylistique et typologique inspiré de l'architecture vernaculaire jurassienne ou germanique, et du «style sapin " pour le programme décoratif extérieur et intérieur. L'architecte n'est-il pas un fervent disciple de Charles l'Eplatennier comme nous pouvons le constater à la lecture architecturale de ses réalisations à Tavannes et à La Chaux-de-Fonds? Chapallaz joue également avec les matériaux: il fait se côtoyer tradition, à travers l'emploi de la pierre de taille, du fer forgé et du bois, et innovation, en intégrant des éléments en béton ou une structure métallique, dans ses constructions. Ce savant mélange de matériaux a donné naissance à d'intéressants bâtiments que nous pouvons rapprocher de ceux construits à la même période dans les montagnes neuchâteloises. En effet, l'atelier d'architecture que Chapallaz se construit à Tavannes ne peut-il pas être directement mis en lien avec la villa Fallet construite à La Chaux-de-Fonds durant la même période? Et l'escalier monumental de la villa Sandoz, l'encorbellement et les murs coupe-vent ne rapellent-t-ils pas des éléments architecturaux de la villa de l'Eplatennier également élevée dans la métropole des montagnes neuchâteloises au début du $\mathrm{XX}^{\mathrm{e}}$ siècle?

\section{L'atelier d'architecture}

De 1905 à 1906, Chapallaz construit une maison qui deviendra son atelier d'architecture (fig. 3 et 4). Elle est située au nord de la villa patronale que la famille Sandoz projette. Il est d'ailleurs fort probable que les deux chantiers soient suivis en parallèle par l'architecte fraîchement arrivé à Tavannes. Cette bâtisse est largement inspirée du Heimatstil par 


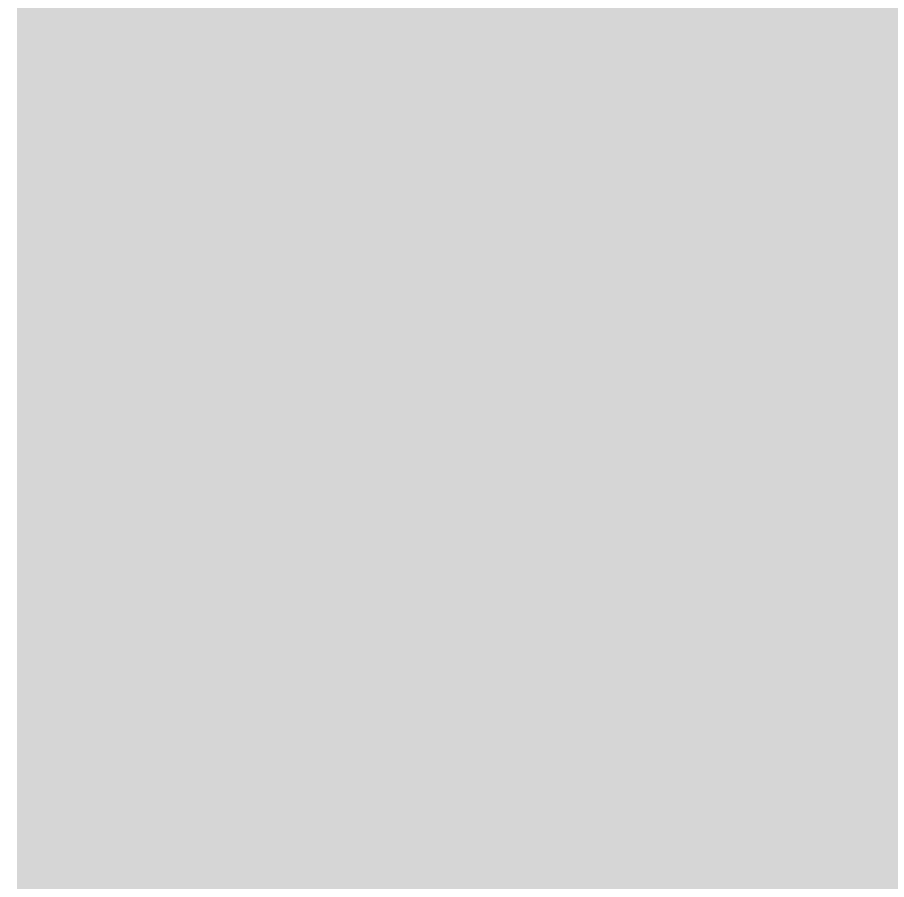

Fig. 3 - Atelier d'architecture, vers 1910.

(Bibliothèque de la ville de La Chaux-de-Fonds. Fonds René Chapallaz).

le traitement rustique de son soubassement en pierre de taille, l'emploi du fer forgé et la massive toiture qui semble directement s'inspirer de la ferme emmentaloise. La maison rappelle la villa Fallet à La Chauxde-Fonds, chantier auquel Chapallaz collabore, ou la Villa Baehler que Chapallaz construit en 1908 lorsqu'il retourne dans les montagnes neuchâteloises. Les nombreux détails architecturaux (un canal de cheminée tourné à 45 degrés, des murs à pans coupés en façade nord, une entrée principale entourée de bow-windows et des fenêtres avec croisillons posés en diagonale) inspirés de la géométrie que forme le sapin des forêts jurassiennes, sont encore visibles aujourd'hui. A l'origine, les tuiles vernissées formaient un motif stylisé également inspiré du conifère des forêts jurassiennes, de même qu'un balcon en façade sud, dont le garde-corps était composé d'éléments géométriques en bois. Sur d'anciennes photographies, ces deux derniers éléments ornementaux sont encore apparents mais ont depuis disparu. Le rapprochement avec les principes décoratifs enseignés à l'Ecole d'art de La Chaux-de-Fonds, dont Chapallaz 
Fig. 4-Atelier d'architecture, 2005.

est un incontestable disciple, est largement présent dans l'œuvre de jeunesse. N'est-il pas officieusement considéré comme «maître de pratique architecturale» pour le cours supérieur au vu de ses contacts avec L'Eplattenier? ${ }^{4}$

4. M. Emery, "Chapallaz versus Jeanneret», p. 23. 
Le programme décoratif intérieur n'est pas laissé au hasard; il se trouve être le reflet de la géométrie que l'on peut déjà saisir à l'extérieur du bâtiment. Les boiseries, les carreaux du carrelage et la porte d'entrée sont traités de manière géométrique, et l'emploi du fer forgé pour les entrées de serrure caractérise l'intervention faite à l'intérieur de la maison. Chapallaz reprendra le même langage stylistique dans les diverses pièces de la villa Sandoz, mais à une échelle supérieure, puisque la décoration intérieure sera effectuée quelques mois plus tard par les élèves de l'Ecole d'art, sous la direction de Charles L'Eplattenier. A peine le chantier de son bureau terminé ou en phase terminale, Chapallaz quitte Tavannes pour un voyage de quelques semaines à travers l'Europe du Nord afin de parfaire ses connaissances architecturales.

\section{Le voyage de 1906}

D'avril à mai 1906, Chapallaz effectue un voyage à travers l'Europe. Il se rend à Darmstadt, Bournville proche de Birmingham, Paris et Nancy. Le choix des étapes de son voyage n'est pas le fruit du hasard mais vraisemblablement dicté par ses diverses lectures. Il est en effet abonné à la revue Le Cottage, revue internationale documentaire illustrée de l'Habitation à bon marché et de l'Art en tout et pour tous publiée à Bruxelles, où l'idéal du Gesamtkunstwerk flirte avec la rusticité et rejoint donc certains principes de l'Ecole d'art de La Chaux-de-Fonds qui s'inspire de la nature et de la flore jurassiennes pour son programme décoratif. En Angleterre, Chapallaz s'intéresse particulièrement à la cité-jardin de Bournville construite par Georges Cadbury et son frère, deux industriels du chocolat. Vers 1900, cette cité-jardin compte environ trois cent treize cottages insérés dans un environnement paysager, des parcs et des espaces publics où se trouvent l'école, les commerces, etc. La dimension sociale et le désir d'offrir aux ouvriers un cadre de vie semblent fortement intéresser Chapallaz, qui mettra en œuvre, en partie, les principes de la cité-jardin à Sonrougeux. 


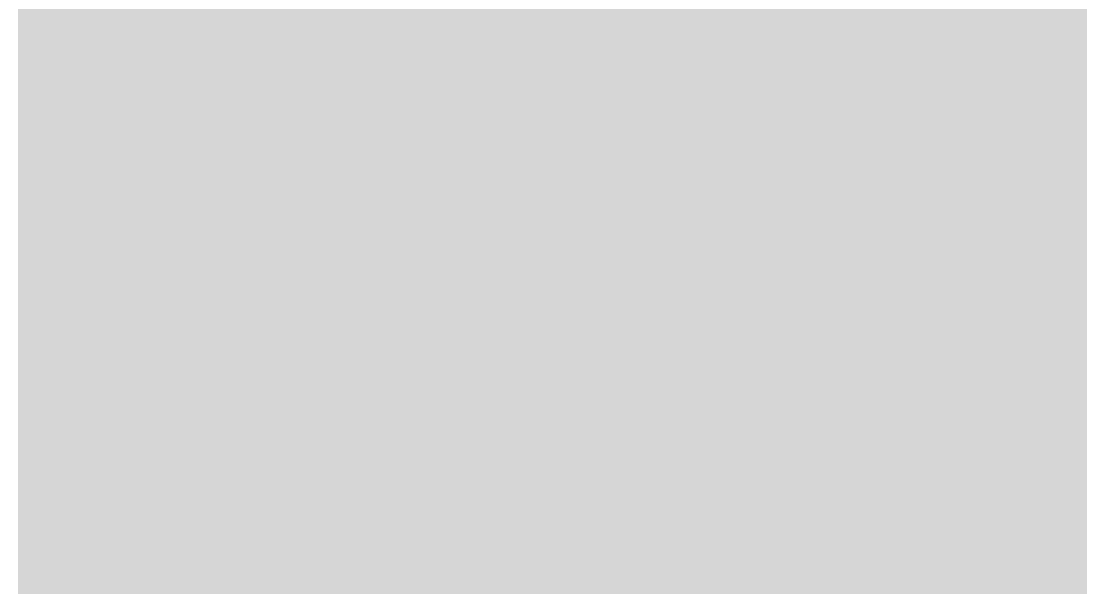

Fig. 5 - Sonrougeux, plan de situation, 1905.

(Bibliothèque de la ville de La Chaux-de-Fonds. Fonds René Chapallaz).

\section{Le lotissement ouvrier de Sonrongeux}

Sonrougeux est une réalisation de première importance pour l'histoire de l'habitat ouvrier dans le Jura bernois. Ce lotissement, construit à partir de 1906, a permis à une dizaine d'ouvriers de devenir propriétaires d'une habitation, certes modeste, dans laquelle un confort standard permettait de vivre dans des conditions de vie de qualité.

Entre septembre et octobre 1905, Chapallaz dessine à l'échelle 1:50 les plans de nombreuses maisons, qu'il «numérote» de $\mathrm{A}$ à $\mathrm{L}$, et deux plans généraux de l'ensemble (fig. 5) ${ }^{5}$. L'ouvrage d'Henry Baudin $\mathrm{La}$ Maison familiale à bon marché acheté en 1904 par Chapallaz sert probablement de référence au projet d'offrir un cadre de vie et un logement de qualité aux ouvriers. La visite de Bournville inspire Sandoz et Chapallaz pour le mode de financement et l'implantation de Sonrougeux.

Dans un premier temps, une coopérative est fondée afin de favoriser l'accession à la propriété à certains employés de la fabrique. Le financement est garanti par la Tavannes Watch Co et l'ouvrier remplace donc son propre employeur. L'intéressé choisit sa maison selon ses désirs et moyens. Différents modèles et grandeurs sont proposés au niveau du

5. Ces plans sont actuellement conservés à la Bibliothèque de la Ville de La Chauxde-Fonds où se trouve également le dépôt des archives de Chapallaz. 


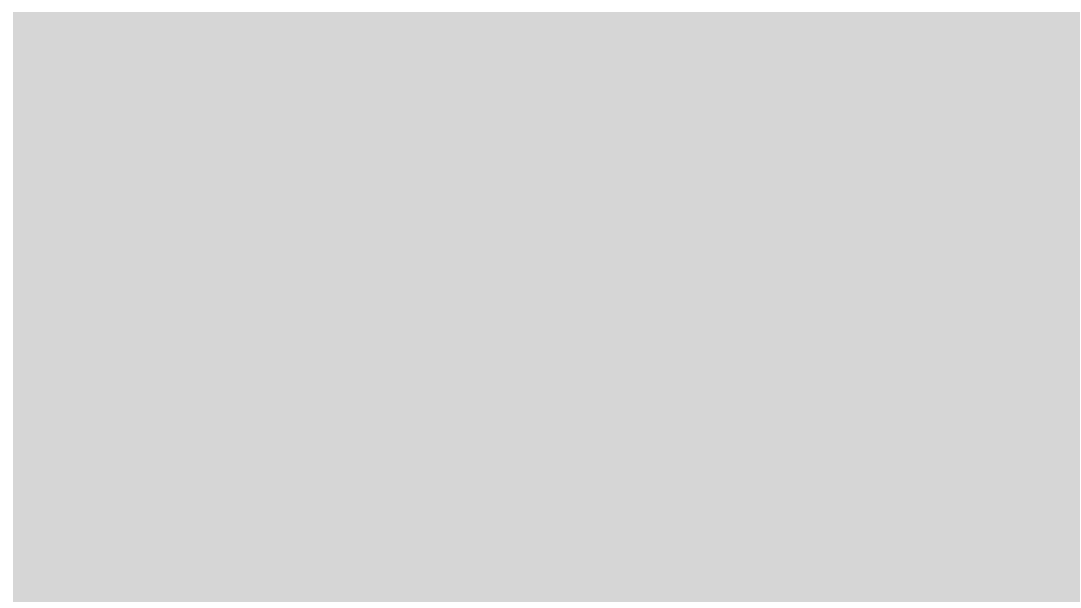

Fig. 6 - Sonrougeux, chantier, vers 1906.

(Bibliothèque de la ville de La Chaux-de-Fonds. Fonds René Chapallaz).

nombre de pièces à disposition. Elles sont d'un prix quasi identique, mais chacune se démarque par une architecture individuelle et fantaisiste. En offrant un cadre de vie agréable à ses chefs d'atelier, le public cible pour la vente, Sandoz met en place, en plus d'une dépendance financière liée au crédit, une fidélité des employés envers l'entreprise.

L'emplacement choisi n'est pas le fruit du hasard. Situé à l'époque à l'extérieur du village, sur le flanc de la colline de Sonrougeux, l'ensemble de cottages surplombe avantageusement Tavannes et profite d'un maximum d'ensoleillement grâce à l'orientation sud-est de la façade principale des maisons. La disposition des villas en deux rangées s'étend d'est en ouest et en retrait l'une de l'autre. Ce choix permet un développement individuel du cadre de vie et une plus grande intimité des propriétaires.

Tous les modèles ont un plan identique au niveau de la distribution des pièces par étage, ces dernières étant de petite dimension. Chaque étage a sa fonction: le sous-sol est réservé aux caves et à la buanderie; le rez-de-chaussée comporte la cuisine, les sanitaires et le séjour ou la grande chambre, comme elle est nommée par l'architecte. C'est l'étage où se déroule la vie de famille et où l'on reçoit ses invités. Au premier étage et sous les combles se trouvent les chambres à coucher et les chambres hautes. Chapallaz s'intéresse également à du mobilier manufacturé à Indianapolis par C. P. McDougall \& Son pour meubler les différents cottages de Sonrougeux mais ne semble pas avoir mené ce projet 


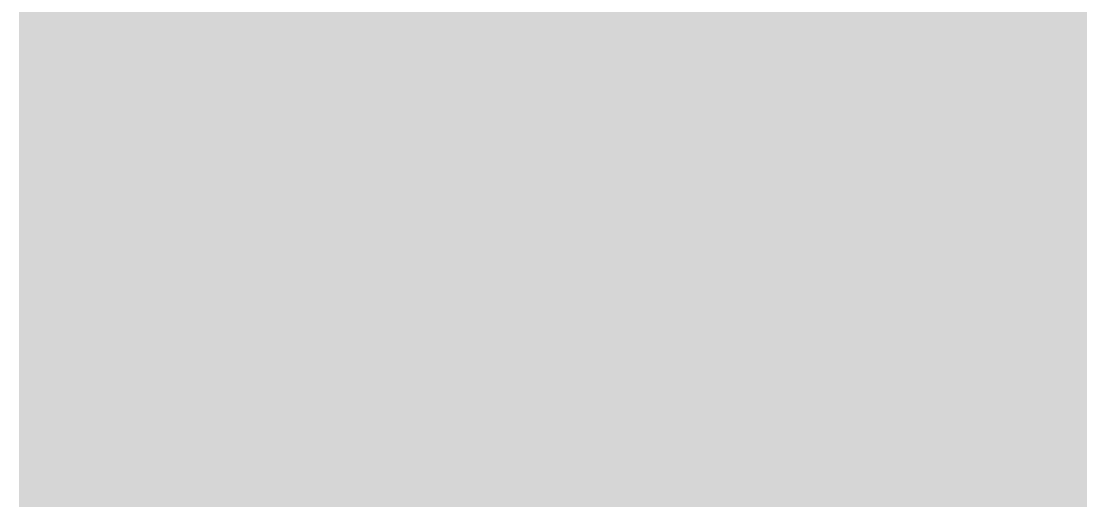

Fig. 7 - Sonrougeux, état en 2001.

à terme. Chaque maison a ses propres annexes qui sont le bûcher ou le poulailler.

Le socle des maisons est constitué d'un appareillage rustique en pierre calcaire, l'élévation est en matériaux traditionnels où le bois prend une place importante (fig. 6). Le choix du béton armé n'est pas retenu alors qu'à la même période, Chapallaz l'emploie pour la construction de la villa Sandoz ainsi que pour son propre bureau. L'aspect extérieur des maisons est relativement simple et ne comporte que peu d'éléments décoratifs. Ces derniers sont parfois présents à travers les menuiseries des balcons ou le colombage utilisé sur quelques réalisations. Ce sont surtout la forme des toitures et les pignons qui attirent le regard et qui donnent son caractère à l'ensemble (fig. 7 et 8). A mi-parcours de sa période à Tavannes, nous pouvons constater la qualité des interventions de Chapallaz dans la petite cité du Jura bernois. Cependant, la réalisation la plus spectaculaire est incontestablement la villa Sandoz dont l'architecte suit les travaux et dont la décoration intérieure sera effectuée par les élèves de l'Ecole d'art de La Chaux-de-Fonds. Encore de nos jours, cette villa conserve une grande partie de son aspect de l'époque.

\section{La villa patronale}

Construite à l'écart du village et orientée sud-ouest, l'ancienne villa Sandoz (fig. 9) est un exemple très intéressant de villa patronale. Fort de l'expérience acquise à travers la construction de la villa Gallet, objet 


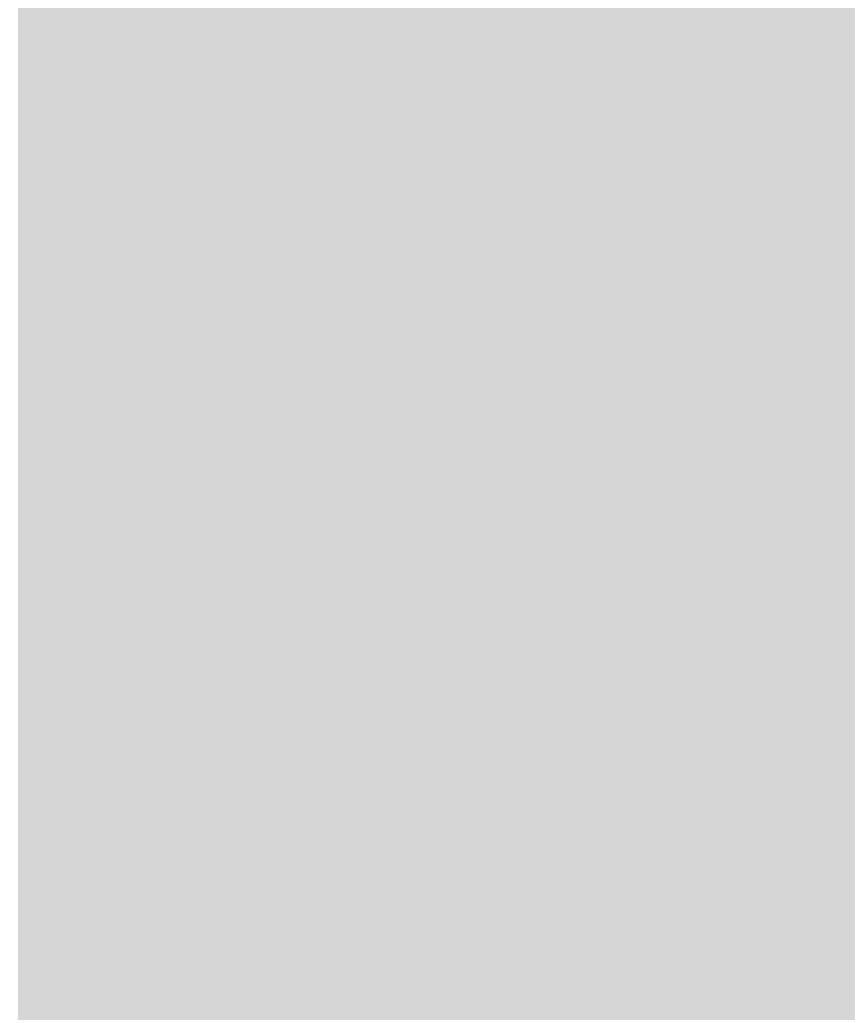

Fig. 8 - Sourougeux, état d'une des maisons en 2001.

prestigieux dans sa réalisation intérieure et extérieure, et de la villa L'Eplattenier, Chapallaz reprend le chantier en cours dès son arrivée à Tavannes. Sur la base des plans des architectes Vuilleumier et Renk, Chapallaz exécute vers 1905-1906 l'enveloppe extérieure de la maison en y intégrant certainement quelques éléments importés de La Chauxde-Fonds. Celle-ci s'inspire de l'architecture vernaculaire jurassienne du XVIII ${ }^{e}$ siècle au niveau de la toiture, de l'emploi de l'encorbellement et des murs coupe-vent en façade sud, éléments architecturaux que l'on retrouve également à la villa de Charles L'Eplattenier construite quelques années auparavant avec la participation de Chapallaz. Le langage stylistique est complété par des éléments de l'architecture classique tels que les colonnes, les doubles absides soulignées par des pilastres et l'escalier principal pour accéder à la maison, qui n'est pas sans rappeler l'architecture palladienne. 
Fig. 9 - Villa Sandoz (premier plan) et atelier d'architecture (second plan), état en 2010.

La villa Sandoz concrétise l'idéal du Gesamtkunstwerk cher à Chapallaz. Il est probablement à l'origine du mandat, que reçoit l'Ecole d'art de La Chaux-de-Fonds, de décorer l'intérieur de la maison. Sandoz rencontre à ce sujet L'Eplattenier à Cernier, comme l'atteste un courrier daté du 18 octobre 1907 envoyé par le fabricant à son fils alors en formation chez Junghans à Schramberg en Allemagne. Il convient de souligner que la villa Sandoz était conçue à la base pour accueillir la famille de Charles Sandoz. Mais cédons la parole à Henri-Frédéric qui semble suivre les travaux pendant l'absence de son fils:

Cet après-midi, nous allons retrouver Isaac Schwob, Mathey-Doret et le peintre L'Eplattenier à Cernier (...). (Nous irons ensuite) à Fontainemelon voir les décors à l'intérieur du temple qui ont été faits par l'Ecole d'art de La Chaux-de-Fonds. M. L'Eplattenier en est le directeur et il désire que Isaac Schwob fasse décorer sa villa par ses élèves et ensuite la nôtre ${ }^{6}$.

6. Ch. Gagnebin-Diacon, La fabrique et le village, p. 128. 
Fig. 10 - Villa Sandoz, Ferronnerie de la porte d'entrée, motif du marronnier, vers 1907-1908.

Quelques mois plus tard, L'Eplattenier et ses élèves, accompagnés peutêtre de Jeanneret, arrivent à Tavannes pour décorer l'intérieur de la maison. Des boiseries sont réalisées, le hall d'entrée et les murs de la plupart des pièces reçoivent un décor peint. Le motif du marronnier se retrouve sur l'imposante ferronnerie qui protège la porte d'entrée vitrée (fig. 10) et sur le garde-corps du balcon du premier étage. Des frises à 
Fig. 11 - Fabrique de la Tavannes Watch Co construite par Chapallaz (second plan), vers 1907 et fabrique plus ancienne, vers 1880 (premier plan).

l'effigie de cet arbre sont peintes dans une chambre du premier étage. Le répertoire décoratif s'inspire de la faune et de la flore de la nature jurassienne comme le préconise L'Eplattenier, fondateur des principes du "style sapin". Nous avons pu le constater, Chapallaz trouve d'intéressantes solutions dans la construction de maisons individuelles mais qu'en est-il du traitement du bâtiment industriel?

\section{La fabrique}

Vers 1907, avant son retour à La Chaux-de-Fonds, Chapallaz entreprend la construction d'une fabrique qui n'est pas intégrée dans le complexe de la Tavannes Watch Co, situé à l'est du village, mais à l'ouest de Tavannes, au nord d'une fabrique plus ancienne que Sandoz avait acquise suite à une faillite. Chapallaz reprend la typologie habituelle de la fabrique de la fin du XIX ${ }^{\mathrm{e}}$ siècle: bâtiment sur deux étages, peu profond mais relativement long. Cependant, il se distancie de la manière usuelle de construire les usines, avec une ossature en bois, par l'emploi d'un système de construction nouveau utilisant du béton et une structure 
métallique (fig. 11). La partie traditionnelle réside dans les tablettes de fenêtres en pierre calcaire. Les allèges murées, en léger retrait, soulignent l'horizontalité du bâtiment qui surprend par sa sobriété. Toujours dans la même frénésie, l'extension de la Tavannes Watch Co est également prévue. Chapallaz dessine des projets d'une usine rayonnante ${ }^{7}$ mais ne réalise pas les travaux, puisqu'à son retour à La Chaux-de-Fonds, il stoppe toutes les initiatives prises auparavant.

Le départ de René Chapallaz ne met pas un terme au programme architectural mis en place par Henri-Frédéric Sandoz. Tavannes continue d'être un énorme chantier tout au long des premières décennies du $\mathrm{XX}^{\mathrm{e}}$ siècle: extension de la Tavannes Watch Co, immeubles locatifs pour les ouvriers, hôtels pour accueillir les clients, restaurants et bien d'autres ouvrages liés directement ou indirectement à l'essor de la fabrique d'horlogerie. Malheureusement, l'entreprise ne va pas survivre à la crise horlogère des années 1970 et la production de montres cesse à Tavannes. De nos jours, le patrimoine industriel est visible et en grande partie conservé et permet une intéressante lecture historique et sociale du développement de l'industrie horlogère dans le Jura bernois.

René Koelliker Historien de l'art

Moutier

7. M. Emery, «Chapallaz versus Jeanneret», p. 26. 


\section{BIBLIOGRAPHIE}

\section{Etudes}

Bieri Thomson, Helen (dir.), Une expérience art nouveau: le style sapin à La Chaux-de-Fonds, Paris, Somogy, 2006.

Crettaz-Stürzel, Elisabeth, Heimatstil. Reformarchitektur in der Schweiz 1896-1914, Frauenfeld, Huber, 2005.

Donzé, Pierre-Yves, Histoire de l'industrie horlogère suisse, de Jacques David à Nicolas Hayek (1850-2000), Neuchâtel, Alphil, 2009.

Emery, Marc, «De la villa à la ville: exposition Chapallaz-Jeanneret, 1900-1920», Archithese, 5 (1982), p. 63.

—, "Chapallaz versus Jeanneret», Archithese, 2 (1983), p. 23-28.

Gagnebin-Diacon, Christine, La fabrique et le village: la Tavannes Watch Co 1890-1918, Porrentruy, Cercle d'études historiques de la Société jurassienne d'Emulation, 1996 ( $2^{\mathrm{e}}$ édition mise à jour en 2006).

Marchand, Gilles, Chapallaz versus Jeanneret: étude comparative des systèmes constructifs, EPFL, Lausanne, 1993.

\section{Crédits photographiques}

Fig. 1:

Archives privées.

Fig. 2-3, 5-6:

Bibliothèque de la ville de La Chaux-de-Fonds. Fonds René Chapallaz.

Fig. 4 et 11 :

Jura bernois tourisme. 
Fig. 7 et 8 :

Service des monuments historiques du canton de Berne.

Fig. 9 et 10 :

René Koelliker. 
